\title{
INTERNET SHOPPING OPTIMIZATION PROBLEM
}

\author{
JACEK BŁAŻEWICZ*,***, MiKhAIL Y. KOVALYOV**, JĘDRZEJ MUSIAŁ*, \\ ANDRZEJ P. URBAŃSKI*, ADAM WOJCIECHOWSKI* \\ * Institute of Computing Science \\ Poznań University of Technology, ul. Piotrowo 2, 60-965 Poznań, Poland \\ e-mail: \{Jacek.Blazewicz, Jędrzej.Musial\}@cs.put.poznan.pl \\ \{Andrzej.Urbanski, Adam.Wojciechowski\}@es.put.poznan.pl \\ ** United Institute of Informatics Problems \\ National Academy of Sciences of Belarus, Surganova 6, 220012 Minsk, Belarus \\ e-mail:kovalyov_my@newman.bas-net.by \\ *** Institute of Bioorganic Chemistry
}

Polish Academy of Sciences, Z. Noskowskiego 12/14, 61-704 Poznań, Poland

\begin{abstract}
A high number of Internet shops makes it difficult for a customer to review manually all the available offers and select optimal outlets for shopping. A partial solution to the problem is brought by price comparators which produce price rankings from collected offers. However, their possibilities are limited to a comparison of offers for a single product requested by the customer. The issue we investigate in this paper is a multiple-item multiple-shop optimization problem, in which total expenses of a customer to buy a given set of items should be minimized over all available offers. In this paper, the Internet Shopping Optimization Problem (ISOP) is defined in a formal way and a proof of its strong NP-hardness is provided. We also describe polynomial time algorithms for special cases of the problem.
\end{abstract}

Keywords: algorithms, computational complexity, combinatorial algorithms, optimization, Internet shopping.

\section{Introduction}

On-line shopping is one of key business activities offered over the Internet. A survey concerning American Internet users behavior published by Pew Internet \& American Life Project in February 2008 (Horrigan, 2008) shows that the population of on-line customers grows rapidly and systematically from year to year. The number of on-line users either buying or searching for products on-line since 2000 has roughly doubled. While in $200022 \%$ of Americans ( $46 \%$ of on-line users) had some experience with buying products in virtual shops, the ratio grew to $39 \%$ in 2003 and reached 49\% (66\% of on-line users) in 2007. The development of on-line shopping is also stimulated by the increasing number of Internet users. E-commerce revenue has grown from $\$ 7.4$ billion in the middle of 2000 to $\$ 34.7$ billion in the third quarter of 2007. A survey concerning the behavior of customers in Poland (Gemius, 2008) confirms the tendency observed in America. Among various business instruments commonly available on-line since 1990s, like auctions (Lee, 1998; Klein, 2000) and (Vulkan, 2003, pp. 149-178), banking and secure payment (Langdon et al., 2000), shopping (Liang and Huang, 1998), electronic libraries (Lesk, 1997), etc., on-line retail remains the service offered by the highest number of providers. A growing number of on-line shops and increased accessibility to customers world-wide due to the use of credit card payment in on-line transactions (Langdon et al., 2000) are key attributes that force strong competition on the market, keep prices low (when compared to off-line shopping) and make more and more customers interested in on-line purchasing (Vulkan, 2003, pp. 22-27). However, a wide choice of on-line shops makes it difficult to manually compare all the offers and choose optimal providers for the required set of products.

A solution of this problem has been supported by software agents (Tolle and Chen, 2000), so-called price comparison sites. The concept of a price comparator is built on the idea of collecting offers of many on-line shops and building a price ranking on a custo- 
mer's request. This approach is commonly accepted by customers and, according to Alexa Rank, popular price comparison services belong to the group of 1000 most viewed sites world-wide: shopping.com 518-th place, nextag.com 533-th place, bizrate.com 600th place, shoplocal . com: 932-th place (site popularity results registered in October 2008, www . al exa . com).

It is worth noting that price ranking built on-line on a customer's request expressed in a text query (product description) is a solution to a specific case of shopping, in which a customer wants to buy a single product. Multiple item shopping is not supported by price comparators available nowadays. Furthermore, price comparison sites, being commercial projects, tend to optimize their incomes from directing customers to particular on-line shops. As a result, price comparison sites play the role of recommender systems (Satzger et al., 2006) which tend to detect a customer's preferences and interests in order to suggest products to buy. A side effect of the problems mentioned above is the loss of customer confidence. To illustrate the optimization process, we would like to consider and assess its benefits. To this end, let us consider an example below. A customer wants to buy five books. The prices of the books and delivery costs in six shops which the customer considers as potential shopping locations are collected in Table 1 .

The customer's goal is to buy all five books at minimum expense. The support from currently available price comparators allows building the customer's basket based on optimal offers for each individual book. The result of the selection process is presented in Table 2

The shopping performed upon price comparator suggestions would not be optimal (total cost: 210) because simple price comparison does not include delivery cost, which grows as the number of shopping locations grows. In order to find the cheapest solution for the ISOP illustrated above, one can perform a complete search of all possible realizations of shopping. The simple example we analyze shows that the optimization process may bring some savings. In our example, the optimal cost of purchase (in Shop 1 and Shop 4) equals 189 (see Table 3). The problem addressed in this paper is to manage a multipleitem shopping list over several shopping locations. The objective is to have all the shopping done at minimum total expense. One should notice that dividing the original shopping list into several sub-lists whose items will be delivered by different providers increases delivery costs. These are counted and paid individually for each package (sub-list) assigned to a specific Internet shop in the optimization process.

In the sequel, we consider the above mentioned Internet shopping optimization problem in a more formal way. In Section 2, a formal definition of the problem is given. In Section 3, we prove that the ISOP is NP-hard in the strong sense and that it is not approximable in polynomial time. In Section 4 we demonstrate that the ISOP is polynomially solvable if the number of products to buy, $n$, or the number of shops, $m$, is a given constant. The paper concludes with a summary of the results and suggestions for future research.

\section{Problem definition}

The notation used throughout this paper is given in Table 4. We study the following problem of Internet shopping. A single buyer looks for a multiset of products $N=\{1, \ldots, n\}$ to buy in $m$ shops. A multiset of available products $N_{l}$, a cost $c_{j l}$ of each product $j \in N_{l}$, and a delivery cost $d_{l}$ of any subset of the products from the shop to the buyer are associated with each shop $l$, $l=1, \ldots, m$. It is assumed that $c_{j l}=\infty$ if $j \notin N_{l}$. The problem is to find a sequence of disjoint selections (or carts) of products $X=\left(X_{1}, \ldots, X_{m}\right)$, which we call a cart sequence, such that $X_{l} \subseteq N_{l}, l=1, \ldots, m$, $\cup_{l=1}^{m} X_{l}=N$, and the total product and delivery cost, denoted by $F(X):=\sum_{l=1}^{m}\left(\delta\left(\left|X_{l}\right|\right) d_{l}+\sum_{j \in X_{l}} c_{j l}\right)$, is minimized. Here $\left|X_{l}\right|$ denotes the cardinality of the multiset $X_{l}$, and $\delta(x)=0$ if $x=0$ and $\delta(x)=1$ if $x>0$. We denote this problem as the ISOP, its optimal solution as $X^{*}$, and its optimal solution value as $F^{*}$.

\section{Strong NP-hardness and inapproximability}

In this section we will analyze the computational complexity of the ISOP. We will demonstrate its strong NPhardness by proving strong NP-completeness of its decision counterpart-Problem P1. The latter has the same input as the ISOP plus an additional parameter $y$, and the question is to determine whether there exists a selection of products with the total cost $F(X) \leq y$.

Theorem 1. The ISOP is NP-hard in the strong sense even if all costs of the available products are equal to zero and all the delivery costs are equal to one.

Proof. We construct a pseudo-polynomial transformation of Problem P1 from the strongly NP-complete problem EXACT Cover BY 3-SETS (X3C), see (Garey and Johnson, 1979).

EXACT COVER BY 3-Sets (X3C) can be defined as follows: Given a family $E=\left\{E_{1}, \ldots, E_{L}\right\}$ of threeelement subsets of the set $K=\{1, \ldots, 3 k\}$, does $E$ contain an exact cover of $K$, i.e., a subfamily $Y \subseteq E$ such that each $j \in K$ belongs to exactly one three-element set in $Y$ ? It is clear that if $Y$ is a solution to $\mathrm{X} 3 \mathrm{C}$, then $|Y|=k$.

Given an instance of $\mathrm{X} 3 \mathrm{C}$, we construct the following instance of Problem P1. There are $m=L$ shops with available products of the sets $N_{l}=E_{l}, l=1, \ldots, L$. The 
Table 1. Prices of books and delivery costs offered by six internet shops.

\begin{tabular}{|c|c|c|c|c|c|c|c|}
\hline Cost & Book $a$ & Book $b$ & Book $c$ & Book $d$ & Book $e$ & Delivery & Total \\
\hline \hline Shop 1 & 18 & 39 & 29 & 48 & 59 & 10 & 203 \\
\hline Shop 2 & 24 & 45 & 23 & 54 & 44 & 15 & 205 \\
\hline Shop 3 & 22 & 45 & 23 & 53 & 53 & 15 & 211 \\
\hline Shop 4 & 28 & 47 & 17 & 57 & 47 & 10 & 206 \\
\hline Shop 5 & 24 & 42 & 24 & 47 & 59 & 10 & 206 \\
\hline Shop 6 & 27 & 48 & 20 & 55 & 53 & 15 & 218 \\
\hline
\end{tabular}

Table 2. Price comparator solution-the result of the selection process.

\begin{tabular}{|c|c|c|c|c|c|c|c|}
\hline & Book $a$ & Book $b$ & Book $c$ & Book $d$ & Book $e$ & Delivery & Total \\
\hline \hline Price & 18 & 39 & 17 & 47 & 44 & 45 & 210 \\
\hline Shop & Shop 1 & Shop 1 & Shop 4 & Shop 5 & Shop 2 & & \\
\hline
\end{tabular}

Table 3. Optimal purchase cost in selected shops.

\begin{tabular}{|c|c|c|c|c|c|c|c|}
\hline & Book $a$ & Book $b$ & Book $c$ & Book $d$ & Book $e$ & Delivery & Total \\
\hline \hline Cost & 18 & 39 & 17 & 48 & 47 & 20 & 189 \\
\hline Shop & Shop 1 & Shop 1 & Shop 4 & Shop 1 & Shop 4 & & \\
\hline
\end{tabular}

buyer would like to purchase the set of products $N=K$. The cost of any product available in any shop is equal to zero $\left(c_{j l}=0, j \in N_{l}, l=1, \ldots, m\right)$, the delivery cost from any shop to the buyer is equal to one $\left(d_{l}=1, l=\right.$ $1, \ldots, m)$, and the threshold value of the criteria is $y=k$. We show that $\mathrm{X} 3 \mathrm{C}$ has a solution if and only if there exists a solution $X$ for the constructed instance of Problem P1 with $F(X) \leq k$. It is easy to see that our transformation is polynomial and pseudo-polynomial at the same time. Therefore, Problem P1 belongs to the class NP.

Let $Y$ be a solution to $\mathrm{X} 3 \mathrm{C}$. Construct a solution to Problem $\mathrm{P} 1$, in which the required products are purchased in $k$ shops determined by their sets of products $N_{l}=E_{l} \in$ $Y$, i.e., $X_{l}=N_{l}$ if $N_{l} \in Y$ and $X_{l}=\emptyset$ if $N_{l} \notin Y$. Since $Y$ is an exact cover of $K$, all the required products are purchased, and the cost of the corresponding solution is $F(X)=k$.

Now assume that there exists a solution $X$ to Problem $\mathrm{P} 1$ with the cost value $F(X) \leq k$. On the one hand, for this solution the number of shops with $X_{l} \neq \phi$ should not exceed $k$ because otherwise $F(X)>k$. On the other hand, the number of these shops should not be less than $k$ because otherwise at least one product $j \in N$ will not be purchased. Therefore, there are exactly $k$ shops with $X_{l} \neq \emptyset$. Since the purchased products form the set $K$, the collection of the shops with $X_{l} \neq \emptyset$ represents a solution to $\mathrm{X} 3 \mathrm{C}$.

We now discuss the approximability of the ISOP. Let us consider its special case, in which the cost of any product in any shop is equal to zero, and the delivery cost from any shop to the buyer is equal to one. This special case is equivalent to the following Minimum SET CoVER problem, see (Crescenzi and Kann, 2008).
Minimum Set Cover: Given a collection $C$ of subsets of a finite set $S$, find a set cover for $S$, i.e., a subset $C^{\prime} \subseteq$ $C$ such that every element in $S$ belongs to at least one member of $C^{\prime}$, which minimizes the cardinality of the set cover, i.e., $\left|C^{\prime}\right|$.

Due to (Raz and Safra, 1997), the problem MinIMUM SET COVER is polynomially non-approximable within the ratio $c \cdot \ln |S|$, for some constant $c>0$. Therefore, the following statement can be formed.

Statement 1. There exists no polynomial $(c \cdot \ln n)$ approximation algorithm for the ISOP, unless $\mathcal{P}=\mathcal{N} \mathcal{P}$.

\section{Polynomial algorithms for special cases}

Notice that the intractability of the ISOP is established under the assumption that both the number of products to buy, $n$, and the number of shops, $m$, are variables. In this section we present two solution approaches for the ISOP, which are polynomial if either $n$ or $m$ is a constant.

The idea of our first algorithm SHOP-ENUM is to enumerate all possible selections of shops containing all the required products, to choose the best cart sequence $X^{(M)}$ for each selection of shops $M, M \subseteq\{1, \ldots, m\}$, to calculate the total product and delivery $\cos$ for each $M$, and, finally, to find a cart sequence $X^{*}$ with the minimum total cost, $F^{*}$.

\section{Algorithm SHOP-ENUM}

Step 1. Set $F^{*}=\infty$ and $X_{l}^{*}=\emptyset, l=1, \ldots, m$.

Step 2. Consider selections of shops to buy all the required products. Each shop $l, l \in\{1, \ldots, m\}$, such that 
Table 4. Problem definition-table of notation.

\begin{tabular}{|c|l|}
\hline Symbol & Explanation \\
\hline \hline $\mathrm{n}$ & number of products \\
\hline $\mathrm{m}$ & number of shops \\
\hline$N_{l}$ & multiset of products available in shop $l$ \\
\hline$c_{j l}$ & cost of each product $j \in N_{l}$ \\
\hline$d_{l}$ & delivery cost for shop $l, l=1, \ldots, m$ \\
\hline$X=\left(X_{1}, \ldots, X_{m}\right)$ & sequence of selections of products in shops $1, \ldots, m$ \\
\hline$F(X)$ & sum of product and delivery costs \\
\hline$\delta(x)$ & $0-1$ indicator function for $x=0$ and $x>0$ \\
\hline$X^{*}$ & optimal sequence of selections of products \\
\hline$F^{*}$ & optimal (minimum) total cost \\
\hline
\end{tabular}

$N_{l}$ is non-empty can be selected or not; therefore, there are at most $2^{m}$ selections. For each selection of shops $M$ perform the following computations. If $M$ does not contain all the required products, then skip considering this selection. Otherwise, do the following: Determine a cart sequence $X^{(M)}=\left(X_{1}^{(M)}, \ldots, X_{m}^{(M)}\right), X_{l}^{(M)} \subseteq N_{l}$, $l=1, \ldots, m$, as follows. For each product $i \in N$, select a shop $l \in M$ in which the cost of product $i$ is lowest. This can be done in constant time if costs $c_{i l}$ are stored for each product $i$ in a heap. Assign product $i$ to the corresponding multiset $X_{l}^{(M)}$. Notice that the assignment strategy can eliminate some of the selected shops. This strategy is optimal for a given selection of shops due to the fact that the total product costs are minimized and the total delivery cost does not exceed the sum of delivery costs for the selected shops. Calculate the total cost, $F\left(X^{(M)}\right)$. If $F\left(X^{(M)}\right)<F^{*}$, re-set $F^{*}:=F\left(X^{(M)}\right)$ and $X^{*}:=X^{(M)}$.

Step 3. Output optimal solution $X^{*}$ with the minimum cost $F^{*}$.

The time complexity of the SHOP-ENUM algorithm is $O\left(n 2^{m}\right)$, which is polynomial (linear) if the number of shops $m$ is a constant.

The idea of our second algorithm, PRODUCTENUM, is to enumerate all possible shop choices for each product. Let $S=\left(S_{1}, \ldots, S_{n}\right)$ be a shop sequence such that $S_{i} \in\{1, \ldots, m\}$ is a shop in which product $i$ will be purchased, $i=1, \ldots, n$. The algorithm determines a shop sequence $S^{*}$ with the minimum total cost, $F^{*}$, of the corresponding cart sequence.

\section{Algorithm PRODUCT-ENUM}

Step 1. Set $F^{*}=\infty$ and $S_{i}^{*}=\emptyset, i=1, \ldots, n$.

Step 2. Consider shop sequences $S=\left(S_{1}, \ldots, S_{n}\right)$ such that product $i \in N$ will be purchased in shop $S_{i}, i=$ $1, \ldots, n$. There are at most $m^{n}$ such sequences. For each sequence $S$, calculate the cost of the corresponding cart sequence, $F(S)$. If $F(S)<F^{*}$, re-set $F^{*}=F(S), S^{*}:=$ $S$ and pass on to considering the next shop sequence.

Step 3. Output optimal shop sequence $S^{*}$ with the minimum total cost $F^{*}$.

The time complexity of the algorithm PRODUCTENUM is $O\left(n m^{n}\right)$, which is polynomial if the number of products $n$ is a constant.

The algorithm PRODUCT-ENUM can be applied in practice if the number of products to buy, $n$, is small and the number of shops having these products, $m$, is large. Alternatively, if the number of shops $m$ is small and the number of the required products $n$ is large, the algorithm SHOP-ENUM can be efficient.

\section{Conclusions}

Internet shopping is often attributed with prices lower than in traditional shops. Another strong advantage of an on-line purchase is a wide choice of alternative retailers which, in general, remain at the same distance from the customer-at least one day for shopping delivery. Postage cost is often non-zero, which makes it reasonable to group purchased products so that each group is ordered in the same shop, and the total purchase and delivery cost is minimized. Changing retail location for the same product can be possible, provided that the customer is guaranteed that the product in a new location is identical to that in an old one. In the case of changing the retail location, the quantity and quality of the product must be identical. However, the total price can change because of different times of delivery, different profit margins, etc. Customers are interested in minimizing the total product and delivery cost. Currently available price comparators can be used for these purposes only occasionally, because they do not provide multiple-item basket optimization.

We introduced the Internet shopping optimization problem and provided a proof of its strong NP-hardness. Furthermore, two polynomial time algorithms for special cases of the ISOP were described. In the future work, we 
intend to derive and experimentally test heuristic approaches for the ISOP to make the suggested approach applicable for solving complex shopping cart optimization problems in on-line applications. The ideas and algorithmic results given in (Musiał and Wojciechowski, 2009) for a simplified version of the ISOP can be generalized and extended for these purposes.

\section{Acknowledgment}

The work was partially supported by the grant from the Ministry of Science and Higher Education no. N519188933. Also, Jacek Błażewicz acknowledges the support of an INRIA Rhone-Alpes grant.

\section{References}

Crescenzi, P. and Kann, V. (2008). A compendium of NP optimization problems, http: //www. nada.kth.se/ viggo/wwwcompendium/

Garey, M. and Johnson, D. (1979). Computers and Intractability: A Guide to the Theory of NP-Completeness, Freeman, New York, NY.

Gemius, S. (2008). E-commerce in Poland, http: //gemius.pl/pl/raporty/2008-06/03

Horrigan, J. (2008), On-line Shopping, Pew Research Center, http://www.pewinternet.org/ /media //Files/Reports/2008 /PIP_Onlinepping.pdf.pdf

Klein, S. (2000). The emergence of auctions on the world wide web, in M. Shaw, R. Blanning, T. Strader and A. Whinston (Eds.), Handbook on Electronic Commerce, SpringerVerlag, Berlin/Heidelberg, pp. 627-645.

Langdon, C., Roghe, F. and Shaw, M. (2000). Consumer mass market on-line payment solutions, in M. Shaw, R. Blanning, T. Strader and A. Whinston (Eds.), Handbook on Electronic Commerce, Springer-Verlag, Berlin/Heidelberg, pp. 273-288.

Lee, H. (1998). Do electronic marketplaces lower the prices of goods?, Communications of the ACM 41(1): 73-80.

Lesk, M. (1997). Practical Digital Libraries: Books, Bytes and Bucks, Morgan Kaufmann Publishers, San Francisco, CA.

Liang, T. and Huang, J. (1998). An empirical study on consumer acceptance of products in electronic markets: A transactional cost model, Decision Support Systems 21(1): 29-43.

Musiał, J. and Wojciechowski, A. (2009). A customer assistance system: Optimizing basket cost, Foundations of Computing and Decision Sciences 34(1): 59-69.

Raz, R. and Safra, S. (1997). A sub-constant error-probability low-degree test, and sub-constant error-probability PCP characterization of NP, 29th Annual ACM Symposium on Theory of Computing, El Paso, TX, USA, pp. 475-484.

Satzger, B. , Endres, M. and Kielssing, W. (2006). A preferencebased recommender system, in K. Bauknecht, B. Pröll and H. Werthner (Eds.), E-Commerce and Web Technologies,
Lecture Notes in Computer Science, Vol. 4082, SpringerVerlag, Berlin/Heidelberg, pp. 31-40.

Tolle, K. and Chen, H. (2000). Intelligent software agents for electronic commerce, in M. Shaw, R. Blanning, T. Strader and A. Whinston (Eds.), Handbook on Electronic Commerce, Springer-Verlag, Berlin/Heidelberg, pp. 265-382.

Vulkan, N. (2003). The Economics of E-Commerce. A Strategic Guide to Understanding and Designing the On-line Marketplace, Princeton University Press, Princeton, NJ

Jacek Błażewicz, born in 1951 (M.Sc. in control engineering: 1974, Ph.D. and D.Sc. in computer science: 1977 and 1980, respectively), is a professor of computer science at the Poznań University of Technology. Presently he is a deputy director of the Institute of Computing Science. His research interests include algorithm design and complexity analysis of algorithms, especially in bioinformatics as well as in scheduling theory. He has published widely in the above fields (over 300 papers) in many outstanding journals. He is the author and co-author of 14 monographs, an editor of the International Series of Handbooks in Information Systems (Springer-Verlag) as well as a member of the editorial boards of several scientific journals. His science citation index reaches 2300 (according to the ISI database). In 1991 he was awarded an EURO Gold Medal for his scientific achievements in the area of operations research. In 2002 he was elected a corresponding member of the Polish Academy of Sciences. In 2006 he was awarded an honorary doctoral degree by the University of Siegen

Mikhail Y. Kovalyov, born in 1959 (Ph.D. and D.Sc. degrees in mathematical cybernetics in 1986 and 1999, respectively, professorial title in computer science: 2004), is a deputy general director of the United Institute of Informatics Problems, National Academy of Sciences of Belarus, and a professor of Belarusian State University. His research interests include combinatorial optimization, scheduling, logistics and bioinformatics. He has authored over 100 publications in refereed scientific journals. His paper co-authored with C.N. Potts is included in the list of 30 best papers published by the European Journal of Operational Research in 1975-2005. His citation $h$-index is 14 according to Scopus and 16 according to Google Scholar. He is a co-author of one monograph and a chapter in a monograph. Mikhail Y. Kovalyov is an associate editor for Omega and the Asia-Pacific Journal of Operational Research, a member of the editorial boards of the European Journal of Operational Research and Computers and Operations Research, a member of the EURO working group on production management and scheduling, and the vice-president of the Byelorussian Operational Research Society.

Jędrzej Musiał, born in 1982 (M.Sc. in computer science from the Poznań University of Technology: 2006) is a Ph.D. student, researcher and lecturer at the Institute of Computer Science of the Poznań University of Technology. His research interests include electronic commerce, algorithm design and combinatorial optimization. He has published several papers. Since 2008 has been a board member of the Polish Information Processing Society (Major Poland Branch).

Andrzej P. Urbański graduated in computer science from the Poznań University of Technology, Poland, and obtained his $\mathrm{PhD}$ in the same field from the Polish Academy of Sciences. Presently he is a researcher and lecturer at the Institute of Computer Science of the Poznań University of Technology. His research includes the fields of computer-aided design, artificial intelligence, and electronic commerce. He has been publishing and presenting papers at many European scientific conferences and has also published articles and books popularizing computer science, especially for children, with some literary setting. Some of his computer programs have been marketed and reviewed in popular media, among others 
by Polish TV networks, Deutsche Rundfunk, BBC, Die Welt, The New York Times, and Canadian CRBC.

Adam Wojciechowski graduated from the Poznań University of Technology (PUT) in 1995 with an M.Sc. in computer science. In 1997/98 he worked at Dublin City University, Ireland. In 2002 he received a Ph.D. degree in computer science/software engineering at the PUT, where he currently works as an assistant professor. His research interests include software engineering, electronic business, optimization issues on financial markets and distant education. He is an author or co-author of over 12 papers published in international scientific journals and international conference proceedings. He has also prepared Polish handbooks for ECDL applicants and published a distant education course for webmasters.

Received: 19 June 2009

Revised: 24 October 2009 